\title{
An analysis of a tau-neutrino hypothesis for the near-horizon cosmic-ray-like events observed by ANITA-IV
}

\section{Remy Prechelt, ${ }^{a, *}$ Stephanie Wissel ${ }^{b}$ and Andrew Romero-Wolf ${ }^{c}$ on behalf of the ANITA Collaboration}

(a complete list of authors can be found at the end of the proceedings)

${ }^{a}$ Dept. of Physics and Astronomy, Univ. of Hawai'i, Mānoa, HI 96822.

${ }^{b}$ Dept. of Physics, Dept. of Astronomy \& Astrophysics, Pennsylvania State University, University Park, PA, 16802.

c Jet Propulsion Laboratory, California Institute for Technology, Pasadena, CA 91109.

E-mail: prechelt@hawaii.edu

We present the results of a simulation of the acceptance of the Antarctic Impulsive Transient Antenna (ANITA) to possible $v_{\tau}$ point source fluxes detected via $\tau$-lepton-induced air showers. This investigation is framed around the detection of four upward-going extensive air shower events observed very close to the horizon in ANITA-IV. These four events as well as the overall diffuse and point source exposure to Earth-skimming $v_{\tau}$ are also compared against published ultrahigh-energy neutrino limits from the Pierre Auger Observatory. We find that while these four events were detected at sky coordinates close to ANITA's maximum $v_{\tau}$ sensitivity and were not simultaneously visible by Auger, the implied fluence necessary for ANITA to observe these events is in tension with limits set by Auger across a wide range of energies and is additionally in tension with ANITA's Askaryan in-ice neutrino channel above $10^{19} \mathrm{eV}$.

$37^{\text {th }}$ International Cosmic Ray Conference (ICRC 2021)

July 12th-23rd, 2021

Online - Berlin, Germany

\footnotetext{
*Presenter
} 


\section{Introduction}

The fourth flight of ANITA (ANITA-IV) observed four below-horizon cosmic-ray-like events that have non-inverted polarity - a $3.2 \sigma$ fluctuation if due to background [1]. Unlike the steeplyupcoming $\left(\sim 30^{\circ}\right.$ below the radio horizon $)$ anomalous events of this type reported in two previous ANITA flights (ANITA-I and ANITA-III), all of the ANITA-IV anomalous events are observed at angles close to the horizon (all $\lesssim 1^{\circ}$ below the horizon).

A possible Standard Model explanation for this new class of near-horizon events is a skimming $v_{\tau}$ interaction in the Earth producing a $\tau$-lepton that escapes into the atmosphere, subsequently decaying and producing an upgoing extensive air shower (EAS). Due to the strong attenuation of UHE neutrinos across the long Earth-crossing chord lengths associated with the two steeply upcoming events, this explanation for the ANITA-I and ANITA-III events is in strong tension with limits set by IceCube and Auger for diffuse and point-like UHE neutrino fluxes [2].

However, ANITA's sensitivity to the $\tau$ EAS channel is highly directional and is maximal near the horizon where it is orders of magnitude larger than for the steeply upgoing angles of the ANITA-I and ANITA-III events. This opens the possibility for an Earth-skimming $v_{\tau}$ explanation for the ANITA-IV events (that occur extremely close to the horizon) by significantly reducing the tension with limits set by Auger and IceCube.

In this work, we update the previous ANITA $v_{\tau}$ EAS sensitivity analysis to estimate the diffuse and point source transient fluxes implied by this new class of events and compare them with the limits imposed by other neutrino observatories.

\subsection{ANITA-IV's Cosmic-Ray-Like Events}

Non-anomalous air shower events observed by ANITA are classified into two categories: (1) direct events that reconstruct above the radio horizon (i.e. ANITA observes the emission directly from the shower at it develops in the atmosphere); and (2) reflected events where ANITA observes the radio emission from air showers after the radio emission has reflected off the surface of Antarctica.

Along with their reconstructed direction, events are also typically classified as direct or reflected by the polarity of the received electric field. Due to the Fresnel reflection coefficient at the air-ice boundary, reflected EAS signals have a completely inverted polarity with respect to the signals observed directly from an EAS without reflection. Over its four flights, ANITA has observed seven direct events and 64 reflected UHECR events [1,3]. The two steeply-upgoing events from ANITA-I and ANITA-III, as well as the four new events observed by ANITA-IV, reconstruct below the horizon but show a non-inverted polarity compared to regular reflected events (i.e. the same polarity as cosmic rays observed from above the horizon).

\section{ANITA's Effective Area to $\tau$-induced Extensive Air Showers}

To simulate ANITA's sensitivity to both diffuse and transient point source fluxes of UHE $\tau$ neutrinos, we have developed a new $v_{\tau}$ simulation code, the Tau Point Source Calculator, or tapioca, that utilizes improved physics and detector models compared to previous ANITA analyses. A flowchart showing the simulation logic, as well as some required data inputs, for this simulation code is shown in Figure 1. 


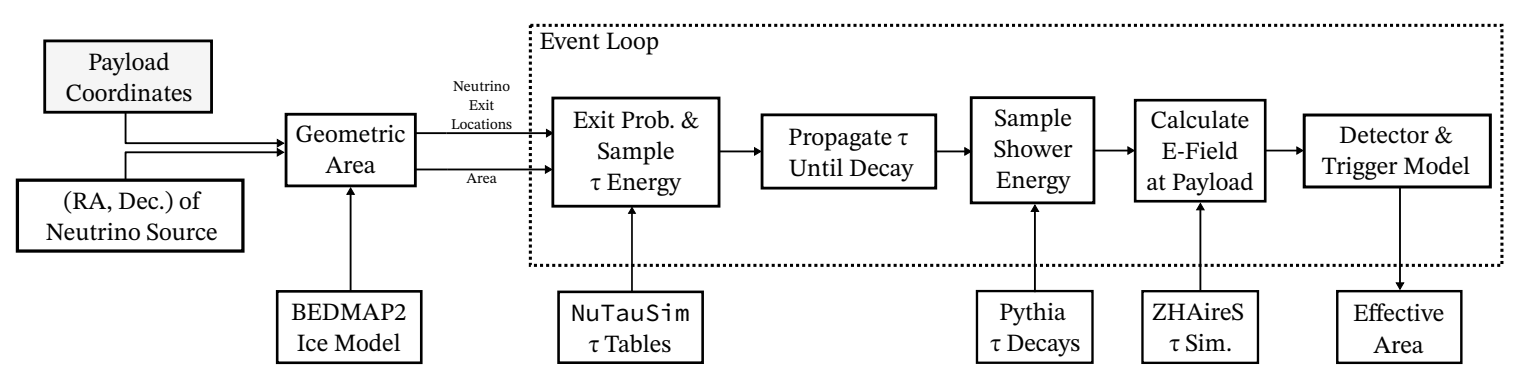

Figure 1: A flowchart showing the top-level logic and event loop of the tapioca simulation code with the external data sources used at each stage.

ANITA's $\tau$-EAS effective area, calculated using tapioca, as a function of the elevation angle of the neutrino source on the sky for a range of energies from $1 \mathrm{EeV}$ to $1000 \mathrm{EeV}$ is shown in Figure 3a. The effective area turns on at several hundred $\mathrm{PeV}$ with a peak effective area of $O\left(1 \mathrm{~km}^{2}\right)$ at the highest energies. The effective area extends $\sim 1^{\circ}$ above the horizon as ANITA observes the radio emission off-axis with respect to the neutrino propagation axis; therefore, an Earth-skimming neutrino from a source slightly above the horizon can still skim the Earth, decay in the air, and be observed $\gtrsim 1^{\circ}$ off-axis.

The peak effective area of ANITA-IV as a function of incident neutrino energy for both the Askaryan channel and the $\tau$ air shower channel is shown in Fig. 3b. The effective area in the $v_{\tau}$ EAS channel exceeds that of the Askaryan channel below $10^{19} \mathrm{eV}$ and significantly lowers ANITA's threshold energy for $v_{\tau}$ detection down to $\sim 0.3 \mathrm{EeV}$. We also show the Pierre Auger Observatory's upgoing $v_{\tau}$ effective area over the same energy range using the published data from [4].

The simulated distribution of neutrino sources detected via the $v_{\tau}$ EAS channel, simulated using tapioca, is shown in Figure 2 along with the most-likely reconstructed direction for each of the four ANITA-IV events. A Kolmogorov-Smirnov test on the reconstructed neutrino source locations against the simulated

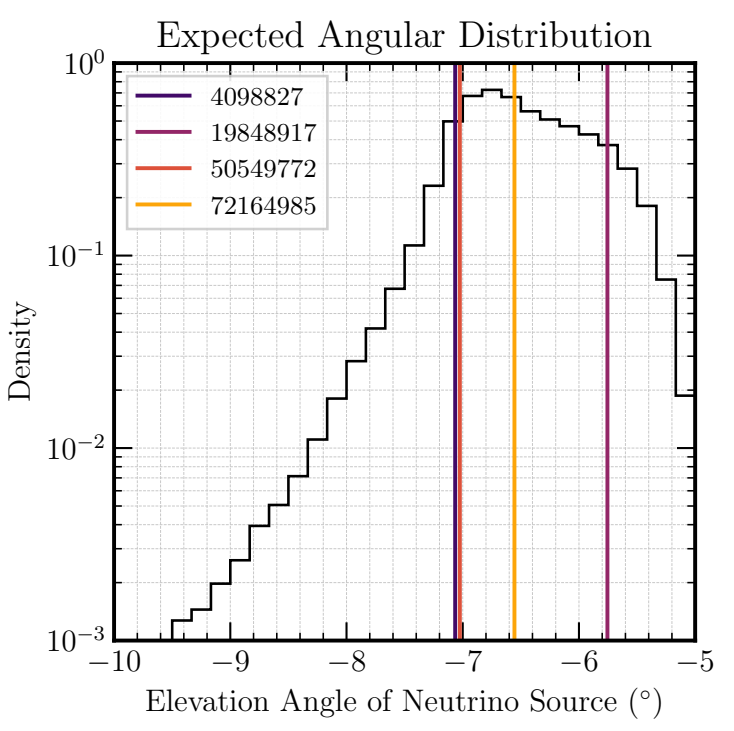

Figure 2: The simulated angular distribution of neutrino sources detected via the $v_{\tau}$ EAS channel compared against the reconstructed elevation angles of each of the four events observed by ANITA-IV. distribution of $v_{\tau}$ events from tapioca found that these events are not inconsistent with the expected angular distribution of detected $v_{\tau}$ sources on the sky.

\section{Reconstructing the Observed Events Under a $v_{\tau}$ Hypothesis}

We use a Bayesian Monte Carlo approach to reconstruct the posterior distributions of $\left(E_{v}, \alpha, \delta\right)$ for each near-horizon event observed by ANITA-IV, where $(\alpha, \delta)$ are the right-ascension and declination of the neutrino source, respectively (under the assumption that these events are from 


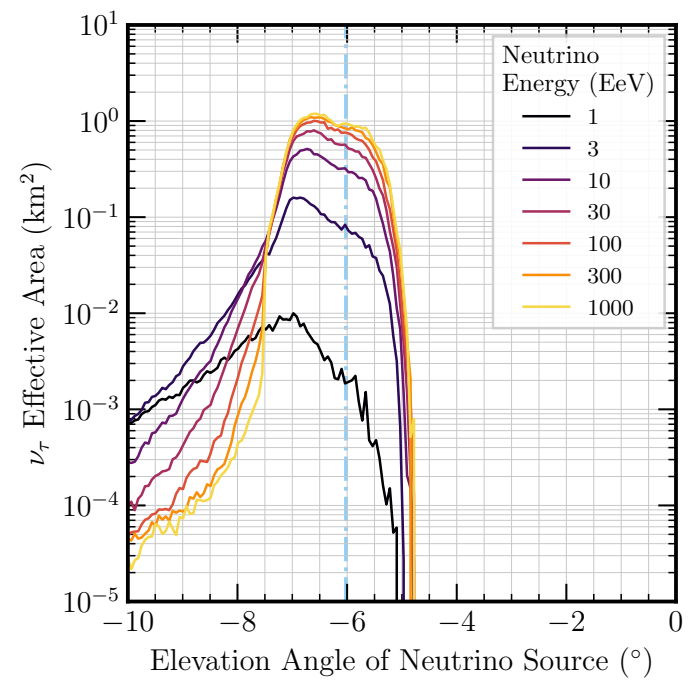

(a) The effective area vs. neutrino source elevation angle for various tau neutrino energies from $1 \mathrm{EeV}$ to $1000 \mathrm{EeV}$. The dashed blue line shows the approximate location of the geometric horizon (averaged over the flight)

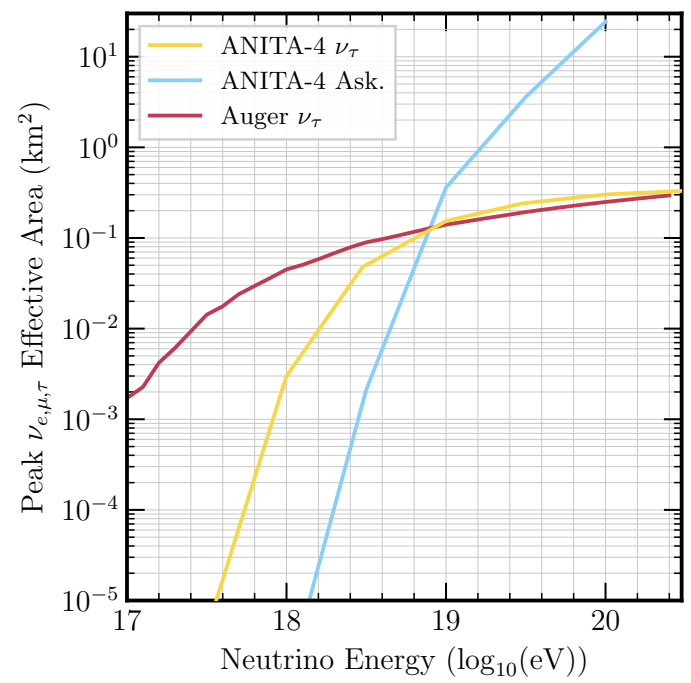

(b) The peak all-flavor (1:1:1) effective area as a function of neutrino energy for the ANITA-IV air shower channel, the ANITA-IV Askaryan channel, and the Pierre Auger Observatory's upgoing $v_{\tau}$ channel.

Figure 3: ANITA-IV's $v_{\tau}$ effective area over elevation angle (left) and over neutrino energy (right).

UHE $v_{\tau}$ 's). In particular, we use the emcee package [5] that implements an affine-invariant ensemble MCMC sampler. For this reconstruction, we use the following likelihood function, implemented in tapioca, that captures the entire process of neutrino emission to the observed radio-frequency signal:

$$
\mathcal{L}\left(E_{\nu}, \alpha, \delta, t\right) \quad \propto \quad A_{g} P_{\text {exit }} P_{\text {decay }} \mathcal{L}_{\theta} \mathcal{L}_{\phi} \mathcal{L}_{\text {waveform }}
$$

where $A_{g}$ is the geometric area for a given $\left(\alpha, \delta, t_{\text {event }}, \vec{x}_{\text {payload }}\right), P_{\text {exit }}$ is the probability that this neutrino generates a $\tau$-lepton that leaves the Earth, $P_{\text {decay }}$ is the probability that the $\tau$-lepton decays before ANITA, $\left(\mathcal{L}_{\theta}, \mathcal{L}_{\phi}\right)$ are Gaussian likelihoods for the observed RF elevation and azimuthal angles, and $\mathcal{L}_{\text {waveform }}$ is a sample-by-sample Gaussian likelihood for the residuals between the forward-modeled (simulated) waveform and the observed waveform for the event.

Since the shape of the neutrino flux at these energies is unobserved, we repeat this likelihood optimization for different priors on the neutrino spectrum. We assume a generic power law neutrino flux shape, $E_{v}^{\gamma}$, between $0.1 \mathrm{EeV}$ and $1000 \mathrm{EeV}$, and reconstruct the neutrino parameters for discrete values $\gamma \in\{-3,-2,-1\}$ to accommodate a range of cosmogenic and astrophysical neutrino models. The most likely neutrino energy depends strongly on the assumed neutrino spectral index, $\gamma$. For example, for Event 72164095 under an assumed $\gamma=-2$, the 50\% quantile in reconstructed neutrino energy is $15.1 \mathrm{EeV}$ with lower and upper $1-\sigma$ quantiles of $7.6 \mathrm{EeV}$ and $42.4 \mathrm{EeV}$, respectively.

The reconstructed neutrino parameters for all of the four events are shown in Table 1 under the various assumptions for $\gamma$. This MCMC, which forward models the entire process from incident neutrino to detection by ANITA, includes uncertainties in the detector models as well as the uncertainty in the observed event parameters. 


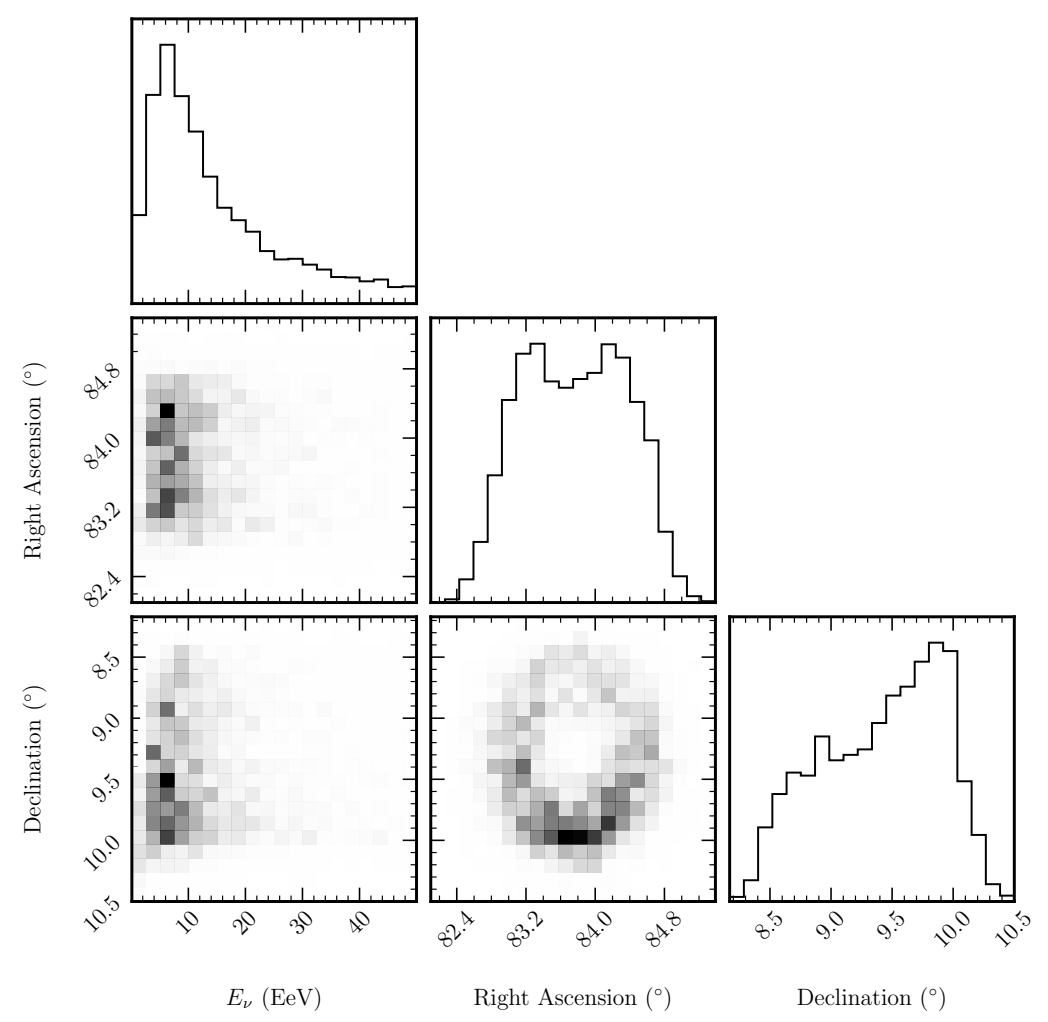

Figure 4: The posterior distributions of the neutrino energy and neutrino source locations, $\left(E_{\nu}, \alpha, \delta\right)$, for Event 72164985 under a $\gamma=-2$ hypothesis as reconstructed by the emcee [5] Markov Chain Monte Carlo using the likelihood function in Equation 1.

\begin{tabular}{c||ccc} 
Event & $E_{v, \gamma=-1}(\mathrm{EeV})$ & $E_{v, \gamma=-2}(\mathrm{EeV})$ & $E_{\nu, \gamma=-3}(\mathrm{EeV})$ \\
\hline \hline 4098827 & $49.8_{-37.7}^{+80.3}$ & $12.5_{-7.4}^{+29.9}$ & $5.2_{-2.5}^{+6.0}$ \\
19848917 & $31.9_{-24.5}^{+76.0}$ & $5.2_{-2.9}^{+11.0}$ & $2.6_{-1.1}^{+3.1}$ \\
50549772 & $45.4_{-34.4}^{+83.4}$ & $8.8_{-4.9}^{+19.5}$ & $4.3_{-2.1}^{+4.8}$ \\
72164985 & $60.3_{-38.2}^{+88.9}$ & $15.1_{-7.6}^{+27.3}$ & $8.9_{-4.5}^{+10.5}$
\end{tabular}

Table 1: The 16\%, 50\%, and 84\% quantiles in neutrino energy $E_{v}$ reconstructed using Eq. 1.

\subsection{Diffuse Flux Limits}

Figure 6a shows the exposure of ANITA-IV to a diffuse $v_{\tau}$ flux via both the Askaryan and upgoing EAS channels (this work). The upgoing EAS channel dominates ANITA's $v_{\tau}$ exposure at energies below $\sim 10^{19} \mathrm{eV}$ above which the Askaryan channel dominates. The significant discrepancy in the total exposure rules out a diffuse isotropic $v_{\tau}$ flux origin for the four ANITA-IV near horizon events under the Standard Model. This is the same conclusion reached for the two steeply upgoing events observed in ANITA-I and ANITA-III [6] and consistent with the expected sensitivity of the three flights [7]. 


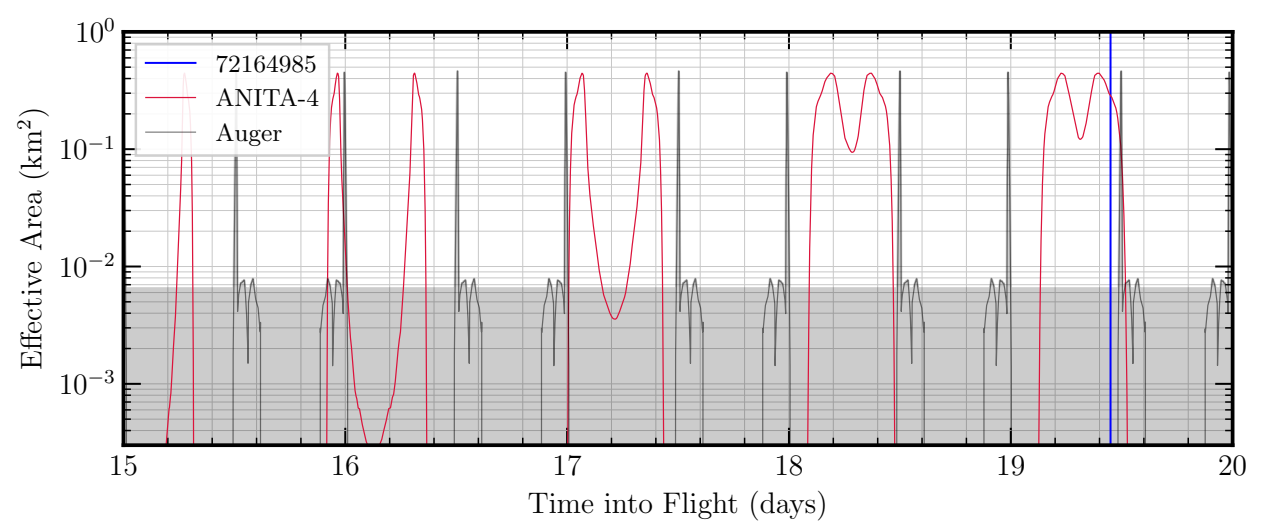

Figure 5: The time evolution of the effective area of ANITA-IV $v_{\tau}$ (pink) and Auger (grey) [4] in the direction of the peak source location corresponding to event 72164985 (see Table 1). Event 72164985 occurred at the time indicated by the blue line.

\subsection{Transient Point Source Limits}

As shown in Figure 5, ANITA's effective area to a given neutrino source location on the sky can be large, but varies significantly as a function of time since the visible portion of the sky changes and ANITA's effective area depends strongly on elevation angle. Therefore, ANITA can set different sensitivity limits on the point source flux depending upon the duration of the transient source and the interplay between the sky location of the source and ANITA's orbit around the continent. The instantaneous single event sensitivity (SES) limit set by ANITA-IV for shortduration ( $\lesssim 15$ minute) and longer duration ( $~ 21$ day) transient neutrino sources occurring at the location of the four observed near-horizon events is shown in Figure $6 \mathrm{~b}$.

\section{Comparison with Other UHE $v$ Observatories}

Under the assumption that ANITA-IV observed 3-4 $v_{\tau}$ events (depending upon background assumptions [1]) from a population of transient neutrino sources, we calculate the flux-modelindependent fluence sensitivity of ANITA's $v_{\tau}$ and Askaryan channels, as well Auger's Earthskimming $v_{\tau}$ channel, for transients of various durations from one second to half-day timescales, as well as for different full-sky transient rates varying from one per-month to several thousand per day. While not an exhaustive search of the parameter space, this covers a representative sample of short- and long-duration transients that are potentially detectable by ANITA without detection by Auger.

For a given transient duration $\Delta T$ and average full-sky event rate $r$, we throw $N \sim \operatorname{Poisson}\left(r T_{\text {auger }}\right)$ random sources on the sky throughout the $T_{\text {auger }} \sim 10$ years of Auger's livetime that we simulate. For each source, we place a box-car time-dependent flux model at the time of each simulated source with the given transient duration, $\Delta T$. We then calculate the total integrated exposure to each of these transients using ANITA-IV's $v_{\tau}$ effective area (this work), ANITA-IV's Askaryan point source effective area, and Auger's upgoing $v_{\tau}$ effective area from [4].

Using the integrated per-source exposure, we calculate the total sensitivity across all sources visible by each experiment assuming that the underlying flux results in ANITA-IV observing $N_{\text {true }}$ 


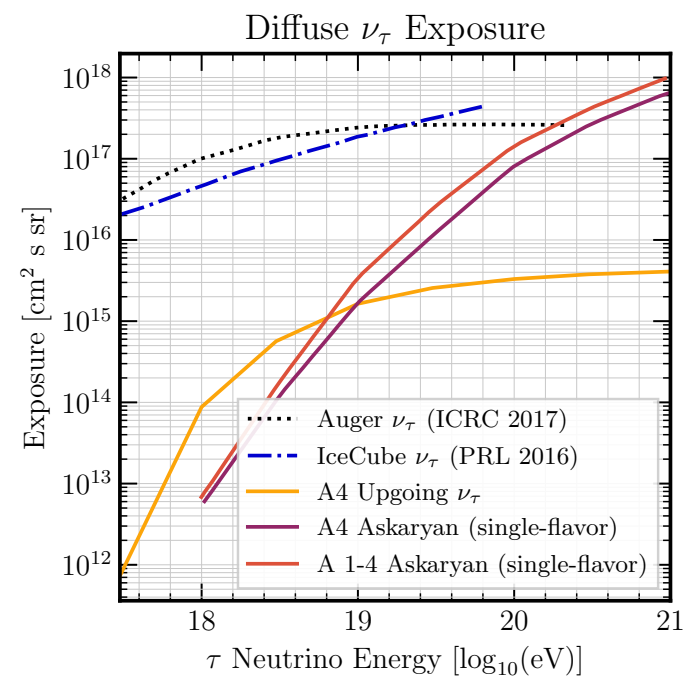

(a) The total integrated exposure to a diffuse UHE $v_{\tau}$ flux for ANITA's Askaryan and $\tau$-EAS channels along with the published exposures of IceCube and Auger

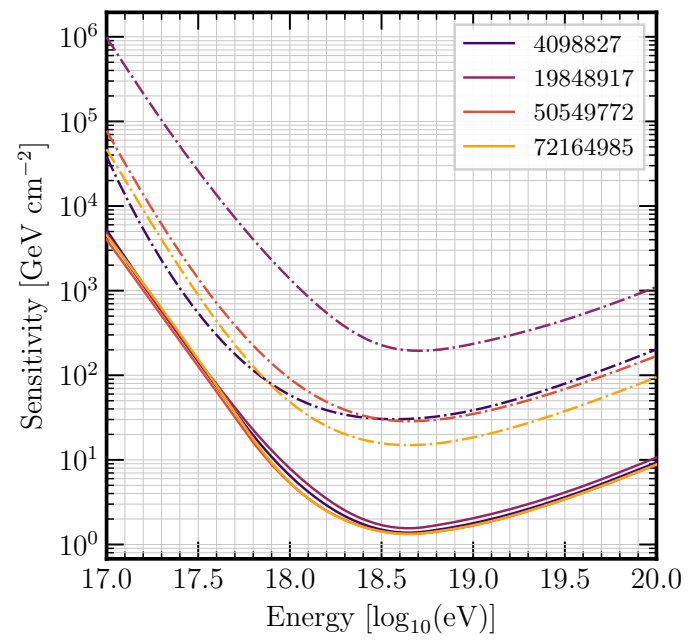

(b) The single-event-sensitivity set by ANITA at the time of each observed near-horizon event for short (§1-min) and longer ( $\gtrsim 1$-day) transients

Figure 6: ANITA-IV's exposure and sensitivity to diffuse and transient UHE neutrino fluxes.

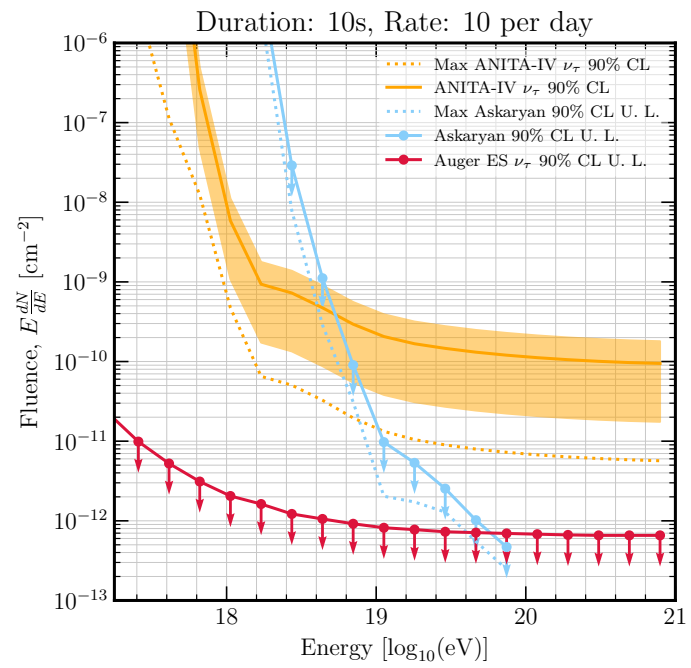

(a) Short-duration transients with a low full-sky event rate.

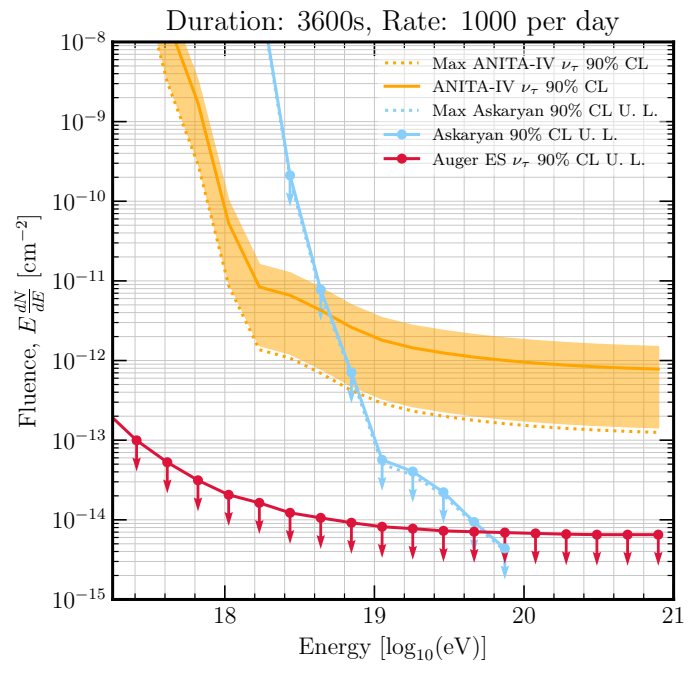

(b) Longer-duration transients with a higher full-sky event rate.

Figure 7: The model independent limits set by ANITA-IV's $\tau$-EAS channel, ANITA-IV's Askaryan channel, and Auger's Earth-skimming $v_{\tau}$ channel for two different combinations of transient rate and duration. 
events, where $N_{\text {true }}$ is sampled from the distribution of the true number of signal events from [1]. We then calculate the corresponding limits on the fluence that would be set by ANITA-IV's Askaryan channel and Auger assuming that no events were detected in either observatory. The model independent limits on the fluence, calculated with this Monte Carlo, is shown in Figure 7 for two representative transient durations and rates.

For all simulated transient durations and full-sky rates, the observation of $\sim 3-4 v_{\tau}$ events is in tension with Auger across the full simulated energy range, and is also in tension with ANITA-IV's Askaryan channel above $\sim 10^{18.8} \mathrm{eV}$. The strength of the fluence limit set by each detector can vary significantly with the transient duration and full-sky transient rate, but Auger always sets a stronger limit than the ANITA-IV $v_{\tau}$ channel for all simulated rates and durations. Above $10^{20} \mathrm{eV}$, ANITA-IV's Askaryan channel is able to set a stronger limit (in $\sim 28$ days) than Auger for all simulated transient durations and full-sky transient rates.

\section{Conclusion}

We have analyzed the plausibility that the upgoing near-horizon ANITA-IV events are explained by $\tau$-lepton-induced extensive air showers from skimming $v_{\tau}$ interactions in the Earth. To achieve this, we have applied detailed models of the $v_{\tau} \rightarrow \tau$ propagation through the Earth, radio emission from air showers, and the ANITA-IV detector. We find that these events are observed at sky locations close to the maximum of the expected distribution of detected $v_{\tau}$ sources. However, there is tension between the $v_{\tau}$ origin of these events and the diffuse and point source $v_{\tau}$ flux limits, both for steady-state and transient sources, set by the Pierre Auger Observatory (calculated by us) as well as ANITA-IV's Askaryan channel above $10^{19} \mathrm{eV}$.

\section{References}

[1] ANITA Collaboration. Unusual Near-Horizon Cosmic-Ray-like Events Observed by ANITAIV. Physical Review Letters, 126(7):071103, February 2021.

[2] ANITA Collaboration. Characteristics of Four Upward-Pointing Cosmic-Ray-like Events Observed with ANITA. Physical Review Letters, 117(7):071101, August 2016.

[3] S. Hoover and et al. Observation of Ultrahigh-Energy Cosmic Rays with the ANITA BalloonBorne Radio Interferometer. Physical Review Letters, 105(15):151101, October 2010.

[4] Auger Collaboration. Limits on point-like sources of ultra-high-energy neutrinos with the Pierre Auger Observatory. Journal of Cosmology and Astroparticle Physics, 2019(11):004, November 2019.

[5] D. Foreman-Mackey, D. W. Hogg, D. Lang, and J. Goodman. emcee: The mcmc hammer. PASP, 125:306-312, 2013.

[6] A. Romero-Wolf, S. A. Wissel, and et al. Comprehensive analysis of anomalous ANITA events disfavors a diffuse tau-neutrino flux origin. Physical Review D, 99(6):063011, March 2019.

[7] Stephanie Wissel, Claire Burch, and et al. Comprehensive estimate of the sensitivity of ANITA to tau neutrinos. PoS, ICRC2019:1034, 2019. 


\section{Full Authors List: ANITA Collaboration}

P. W. Gorham ${ }^{1}$, P. Allison ${ }^{4}$, O. Banerjee ${ }^{4}$, L. Batten ${ }^{5}$, J. J. Beatty ${ }^{4}$, K. Belov ${ }^{3}$, D. Z. Besson ${ }^{6,7}$, W. R. Binns ${ }^{8}$, V. Bugaev ${ }^{8}$, P. Cao ${ }^{9}$, C. H. Chen ${ }^{10}$, P. Chen ${ }^{10}$, Y. Chen ${ }^{10}$, J. M. Clem ${ }^{9}$, A. Connolly ${ }^{4}$, L. Cremonesi ${ }^{11}$, B. Dailey ${ }^{4}$, C. Deaconu ${ }^{12}$, P. F. Dowkontt ${ }^{8}$, B. D. Fox ${ }^{1}$,

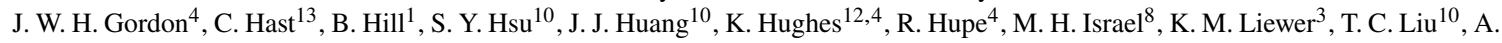
B. Ludwig ${ }^{12}$, L. Macchiarulo ${ }^{1}$, S. Matsuno ${ }^{1}$, K. McBride ${ }^{4}$, C. Miki ${ }^{1}$, K. Mulrey ${ }^{9}$, J. Nam ${ }^{10}$, C. Naudet ${ }^{3}$, R. J. Nichol ${ }^{5}$, A. Novikov ${ }^{6,7}$, E. Oberla ${ }^{12}$, S. Prohira ${ }^{4,6}$, R. Prechelt ${ }^{1}$, B. F. Rauch ${ }^{8}$, J. Ripa ${ }^{10}$, J. M. Roberts ${ }^{1}$, A. Romero-Wolf ${ }^{3}$, B. Rotter ${ }^{1}$, J. W. Russell ${ }^{1}$, D. Saltzberg $^{5}$, D. Seckel ${ }^{9}$, H. Schoorlemmer ${ }^{1}$, J. Shiao ${ }^{10}$, S. Stafford ${ }^{4}$, J. Stockham ${ }^{6}$, M. Stockham ${ }^{6}$, B. Strutt ${ }^{5}$, M. S. Sutherland ${ }^{4}$, G. S.

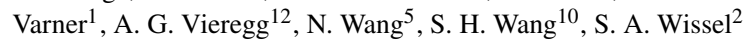

${ }^{12}$ Deptartment of Physics, Enrico Fermi Inst., Kavli Inst. for Cosmological Physics, Univ. of Chicago, Chicago, IL 60637., ${ }^{5}$ Dept. of Physics and Astronomy, Univ. of California, Los Angeles, Los Angeles, CA 90095., ${ }^{4}$ Dept. of Physics, Center for Cosmology and AstroParticle Physics, Ohio State Univ., Columbus, OH 43210., ${ }^{1}$ Dept. of Physics and Astronomy, Univ. of Hawaii, Manoa, HI 96822., ${ }^{10}$ Dept. of Physics, Grad. Inst. of Astrophys., Leung Center for Cosmology and Particle Astrophysics, National Taiwan University, Taipei, Taiwan., ${ }^{6}$ Dept. of Physics and Astronomy, Univ. of Kansas, Lawrence, KS 66045., ${ }^{8}$ Dept. of Physics, McDonnell Center for the Space Sciences, Washington Univ. in St. Louis, MO 63130., ${ }^{13}$ SLAC National Accelerator Laboratory, Menlo Park, CA, 94025. ${ }^{9}$ Dept. of Physics, Univ. of Delaware, Newark, DE 19716., ${ }^{5}$ Dept. of Physics and Astronomy, University College London, London, United Kingdom., ${ }^{11}$ School. of Physics and Astronomy, Queen Mary University of London, London, United Kingdom., ${ }^{3}$ Jet Propulsion Laboratory, California Institute for Technology, Pasadena, CA 91109., ${ }^{2}$ Physics Dept., Pennsylvania State Univ., State College, PA 16802., ${ }^{7}$ Moscow Engineering Physics Institute, Moscow, Russia. 